\title{
A study of the long-term flux density variation of PSRs B0329+54 and B1508+55
}

\author{
A. Esamdin ${ }^{1,2}$, A. Z. Zhou ${ }^{3}$, and X. J. Wu ${ }^{1,2}$ \\ 1 Department of Astronomy, Peking University, Beijing 100871, PR China \\ e-mail: aliyi@ms.xjb.ac.cn \\ 2 Urumqi Observatory,the National Astronomical Observatories, the Chinese Academy of Sciences, Urumqi 830011, PR China \\ 3 Shanghai Astronomical Observatory, Chinese Academy of Sciences, Shanghai 200030, PR China
}

Received 22 March 2004 / Accepted 25 May 2004

\begin{abstract}
We have observed the flux densities of PSRs B0329+54 and B1508+55 for 460 days at $327 \mathrm{MHz}$ using the $25 \mathrm{~m}$ telescope of NAOC Urumqi Observatory. The flux density time series and the corresponding structure functions are presented. Our observational results at $327 \mathrm{MHz}$ and other observations at $74 \mathrm{MHz}$ and $610 \mathrm{MHz}$ are compared with the predictions of the refractive scintillation theory. The frequency dependence of the refractive scintillation parameters for PSR B0329+54 has been investigated. The results show that the long-term flux density modulations of PSR B0329+54 at the three frequencies are best described by a Kolmogorov spectrum for electron density inhomogeneities. For PSR B1508+55, we suggest that the spectrum of the electron density fluctuation has a power exponent $\beta<4$.
\end{abstract}

Key words. stars: pulsars: individual: PSR B0329+54, PSR B1508+55 - ISM: structure - plasmas - turbulence

\section{Introduction}

Two kinds of strong scintillation, diffractive interstellar scintillation (DISS) and refractive interstellar scintillation (RISS), emerged in pulsar radio astronomy. The DISS effects, in which the intensity varies with characteristic timescales of minutes, are believed to be due to the scattering of small spatial scale density fluctuations $\left(10^{6}-10^{8} \mathrm{~m}\right)$ in the interstellar medium (ISM). DISS effects are narrowband (about $100 \mathrm{kHz}$ )in frequency. The long-term flux density variations with characteristic timescales of days to months are attributed to the RISS effects, which are produced by large-scale $\left(10^{10}-10^{12} \mathrm{~m}\right)$ irregularities in the ISM (Sieber 1982; Rickett et al. 1984). The RISS effects are generally broadband in frequency. In addition to the long-term flux density variations, the refractive effects include timing perturbations, modulations of DISS observables, drifting bands in dynamic scintillation spectra, and image wandering. Recently, Bhat et al. (1999a,b,c) investigated the combined effects due to diffractive and refractive interstellar scintillations and suggested the necessity of more comprehensive theoretical treatments to describe refractive fluctuations. Unlike DISS effects, there are several aspects of RISS phenomena that remain to be well understood (e.g. Rickett 1990; Cordes \& Rickett 1998).

Observations of DISS and RISS effects are used to obtain crucial information about the distribution of the electron density along the line of sight. The electron density fluctuations are thought to arise from turbulence and can be characterized by their spatial wavenumber spectrum (e.g. Rickett 1990;
Armstrong et al. 1995; Lambert \& Rickett 1999). According to current understanding, the three-dimensional electron density spectrum is considered to be a simple power-law form $P_{\mathrm{Ne}}(k)=C_{\mathrm{Ne}}^{2} k^{-\beta}$ in the range $k_{\text {outer }} \ll k \ll k_{\text {inner }}$, where $k$ is the spatial wavenumber, which is inversely related to the length scale of electron density fluctuation. $k_{\text {outer }}$ and $k_{\text {inner }}$ correspond to outer and inner cut-offs in scales of the spectrum, respectively. The power-law index $\beta$ is thought to be in the range $3<\beta<5$, and the value for a simple Kolmogorov turbulence is $\beta=11 / 3$. The quantity $C_{\mathrm{Ne}}^{2}$ is a measure of the electron density fluctuation, which gives rise to scattering along a particular line of sight. Knowledge of the spectrum would provide valuable insight into the physics of the interstellar medium. Kolmogorov turbulence results if there is a cascade of energy from a large outer scale $\left(1 / k_{\text {outer }}\right)$ downward in size without energy loss until the energy is dissipated as heat at a small inner scale $\left(1 / k_{\text {inner }}\right)$. A steep spectrum $(\beta>4)$ model (Blandford \& Narayan 1985) and an inner scale model (Coles et al. 1987) were developed to explain enhanced refractive scintillation (e.g. Cordes \& Wolszczan 1986; Rickett et al. 1997). Spectra having $\beta>4$ can result from a superposition of large objects, like interstellar clouds and do not have turbulence connotations. The inner scale model includes a cutoff at an intermediate scale at which the turbulent energy is dissipated, and predicts increasing levels of refractive scintillations with increasing inner scale. However, the exact form of the spectrum and the spatial-scale range over which such a spectrum is valid are still open to discussion. 
The structure-function analyses are extensively used to obtain refractive scintillation parameters. Structure functions are easy to interpret for uniformly sampled data with missing data points, and they are unbiased estimators of the fluctuation power in the absence of a well determined mean. However, it needs long observation duration $\left(T_{\mathrm{obs}}\right)$ and a nearly daily sampling interval to observe significant refractive modulation in many pulsars. Rickett \& Lyne (1990) reported 550 day observations of the Crab pulsar at $610 \mathrm{MHz}$ using a $13 \mathrm{~m}$ antenna and established much of the analysis procedure followed in later studies. Gupta et al. (1993, hereafter GRC93) presented 400 days of flux density monitoring for nine nearby pulsars at $74 \mathrm{MHz}$. LaBrecque et al. (1994) studied 20 pulsars at $430 \mathrm{MHz}$ observed over a $4 \mathrm{yr}$ span. Bhat et al. (1999a) presented results of a project of dynamic-scintillation-spectra monitoring of 18 pulsars at $327 \mathrm{MHz}$ during a $3 \mathrm{yr}$ period. They studied refractive effects in pulsar scintillation and obtained reliable estimates of diffractive scintillation properties. Recently, Stinebring et al. (2000, hereafter S2000) presented results of flux density monitoring of 21 pulsars at $610 \mathrm{MHz}$ on a daily basis over a period of five years. However, the refractive scintillation time scale $T_{\mathrm{r}}$ (i.e. the time lag at which the structure function reaches half its saturation value), has not yet been precisely obtained for some pulsars observed because the length of the observing span is still shorter than the longest time scales in the RISS modulation.

Long-term flux density monitoring and the subsequent structure function analysis at several frequencies are important to test the scintillation model and to constrain the parameters of interstellar medium (Zhou et al. 2003a,b). In this paper, we report a flux density monitoring of PSRs B0329+54 and $\mathrm{B} 1508+55$ at $327 \mathrm{MHz}$ over a period of 460 days using the $25 \mathrm{~m}$ radio telescope of NAOC Urumqi Observatory. Our observational procedure is introduced in Sect. 2. In Sect. 3, we present the time series along with the observed structure functions. The observed modulation parameters are compared with the theoretical predictions in Sect. 4. Discussion and some conclusions of this work are presented in Sect. 5.

\section{Observations}

The long-term flux density monitoring of PSRs B0329+54 and B1508+55 was performed from April 15, 1999 to July 7, 2000 using the Urumqi $25-\mathrm{m}$ radio telescope at $327 \mathrm{MHz}$. The pulsar-monitoring program ran when the telescope was not being used for VLBI observations.

Linearly polarized signals were fed into a single-channel pulsar back-end with a bandwidth of $2 \mathrm{MHz}$, and the signals were square-law detected and stored on disk for analysis. A total of 50-60 min were spent in obtaining each data file with a sampling interval of $2 \mathrm{~ms}$. During each pulsar observation, we recorded signal from cold sky and a noise calibration source, which was injected at the front end, before and after the telescope was pointed at the pulsar observed. So in each data set, there are two calibration scans with a fixed time span just before and after the pulsar signals as a primary power reference for off-line pulsar intensity calibration.
Before each pulsar observation, a continuum source of known flux density intensity at a declination close to that of the pulsar was observed for gain calibration. The data from the calibrator were acquired for 3 min duration, and an equal stretch of data was acquired by pointing the telescope at the cold sky. When the telescope was pointed at the cold sky, the noise calibration source was turned on for one minute. Pulsar flux densities were then computed using the known gain characteristics of the instrument. The data from all calibrations taken on a given day were also used to monitor the stability of the telescope gain during the observing periods. Interference and the disparity between pulsar and continuum source flux densities have affected the accuracy of our absolute calibration. We should note that both pulsars are highly linearly polarized, therefore depending on our observational bandwidth, time span of each observation and the rotation measures of the two pulsars only a fraction of the total intensity is measured by using one linearly-polarized single observations on the flux density measurements.

\section{Data reduction and results}

We present in Fig. 1 the time series of the flux density for PSRs B $0329+54$ (top right) and B1508+55 (bottom right). The flux densities plotted have been divided by the mean value for that time series, and the neighboring points have been connected by solid line to guide the eye. A total of 215 observations were conducted for each pulsar over a period of 460 days. As shown in the two time series of Fig. 1, there are two large gaps in each time series; the time-span of one gap is 37 days, and that of the other is 59 days. Our observations in the remaining 361 days are interrupted by VLBI observations and the regular maintenance of the telescope.

Corresponding structure functions for the two pulsars are shown on log-log scales in Fig. 1 (left two diagrams). We obtained the structure functions following the method described by Rickett \& Lyne (1990). The first order of the structure function of the flux density is

$D(\tau)=\frac{1}{\langle F\rangle^{2}} \sum_{j=1}^{n} \frac{\left(I_{j}-I_{j+\tau}\right)^{2}}{W_{\tau}}$

where $\langle F\rangle$ is the mean of the flux density time series, and $W_{\tau}$ is the total number of data pairs with lag $\tau$ days. $I_{j}$ is the value of the flux density on the $j$ th day. These structure functions displayed in Fig. 1 have been corrected for the presence of uncorrelated noise as discussed by S2000.

A typical structure function displays three distinct regimes: a noise regime at small lags, a structure regime characterized by a linear slope in a log-log plot, and a saturation regime where the structure function flattens out (Stinebring \& Condon 1990). For time lags much shorter than the shortest timescale present in random process, the structure function shows a noise regime. For time lags much longer than the timescale present in a stationary random process, the structure function will reach a saturation value $D(\infty)$.

We use the structure functions to calculate measured refractive parameters, refractive timescale $T_{\mathrm{r}}$, modulation index $m_{\mathrm{r}}$ 

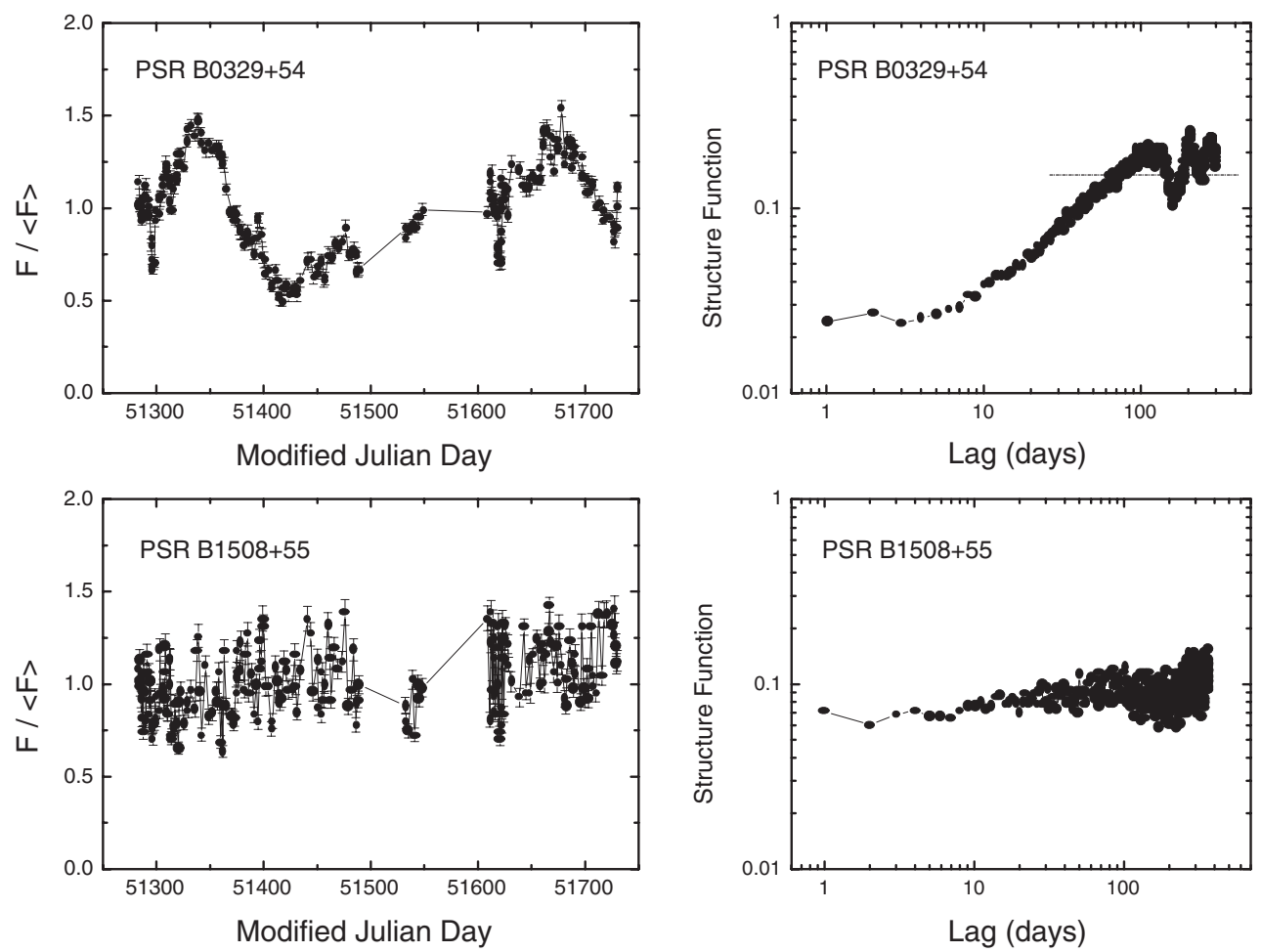

Fig. 1. Flux density time series and structure functions for PSRs B0329+54 and B 1508+55 at $327 \mathrm{MHz}$. Left two diagrams show the flux density modulation, the error bars span \pm 2 standard errors. The flux densities plotted have been divided by the mean value for each time series. The two diagrams on the right present the structure functions on logarithmic scales. The solid line in the top-left diagram indicates the saturation level of structure function of PSR B0329+54.

and logarithmic slope $\gamma$ of the structure regime. Assuming the saturation value of structure function to be $D(\infty)$, the time scale of the flux density variation $T_{\mathrm{r}}$ is determined by the relation $D\left(T_{\mathrm{r}}\right)=D(\infty) / 2$ and the modulation index is defined as $m_{\mathrm{r}}=(D(\infty) / 2)^{1 / 2}$. We measured the logarithmic slope $\gamma$ of the structure regime by following the method described by Kaspi \& Stinebring (1992). The errors of the mean flux density $\langle F\rangle$, the saturation value $D(\infty)$ and the modulation index can be estimated as

$\frac{\delta F}{F} \approx \frac{m_{\mathrm{r}}}{\sqrt{\frac{T_{\mathrm{obs}}}{T_{\mathrm{r}}}}}$

$\frac{\delta D(\infty)}{D(\infty)} \approx \sqrt{\frac{2 T_{\mathrm{r}}}{T_{\mathrm{obs}}}}$

$\frac{\delta m_{\mathrm{r}}}{m_{\mathrm{r}}} \approx \sqrt{\frac{T_{\mathrm{r}}}{T_{\mathrm{obs}}}}\left(m_{\mathrm{r}}+\frac{1}{\sqrt{2}}\right)$

and the estimated error on the refractive time scale is then found from the lags at $(D(\infty)+\delta D(\infty)) / 2$ and $(D(\infty)-$ $\delta D(\infty)) / 2$ (Kaspi \& Stinebring 1992). We present the measured flux densities, modulation indices, refractive timescales, and the logarithmic slope of the structure functions at $327 \mathrm{MHz}$ in Table 1.

As shown in Fig. 1, the structure function of PSR B0329+54 has clearly saturated, presenting three distinct regimes. The structure function rises to a maximum at a lag of 115 days and starts to oscillate indicating an approach to saturation. The saturation level of the structure function $D(\infty)$ is 0.15 . The measured $m_{\mathrm{r}}$ is $0.27 \pm 0.07, T_{\mathrm{r}}$ is $31 \pm 14$ days and the logarithmic slope is $0.7 \pm 0.1$.

The signal to noise ratio for the pulsar is less than that for PSR B0329+54 and is responsible for the relatively noisy appearance of its structure function. The structure function of PSR B1508+55 appears to be in the noise regime (Fig. 1) and does not show the structure and saturation regimes that are present in fully developed structure function. There are two possibilities. One is that the refractive time scale of the pulsar is very small. In this case, the refractive time scale may be less than the minimum observable time scales of our observations. Another possibility is that the refractive time scale may be extremely long, much longer than the duration of our observation.

To show the frequency dependence of refractive parameters of both pulsars, our observational results at $327 \mathrm{MHz}$ are compared with those of other observations at $74 \mathrm{MHz}$ (GRC93) and $610 \mathrm{MHz}$ (S2000; Kaspi \& Stinbring 1992) in Table 1. GRC93 made daily observations of the flux densities of both pulsars using a large phased-array at $74 \mathrm{MHz}$ for 400 days, and estimated refractive scintillation parameters for the variations. The observational parameters for PSR B1508+55 at $610 \mathrm{MHz}$ in Table 1 are taken from Kaspi \& Stingbring (1992), and those for PSR B0329+54 at $610 \mathrm{MHz}$ are from S2000.

\section{Comparison between observations and theory}

Theoretical values of RISS can be predicted by current pulsar scintillation theory using measured parameters of DISS. 
Table 1. Observational and predicted RISS parameters at three frequencies.

\begin{tabular}{|c|c|c|c|c|c|c|c|c|}
\hline \multirow[b]{2}{*}{ PSR B } & \multirow[b]{2}{*}{$v_{\mathrm{obs}}$} & \multirow[b]{2}{*}{$S_{\text {obs }}$} & \multicolumn{3}{|c|}{ Observed parameters } & \multirow[b]{2}{*}{ Notes } & \multicolumn{2}{|c|}{ Predicted parameters } \\
\hline & & & $m_{\mathrm{r}}$ & $T_{\mathrm{r}}$ & $\gamma$ & & $m_{\mathrm{r}, \mathrm{p}}$ & $T_{\mathrm{r}, \mathrm{p}}$ \\
\hline & $(\mathrm{MHz})$ & $(\mathrm{mJy})$ & & (days) & & & & (days) \\
\hline \multirow[t]{3}{*}{$0329+54$} & 610 & & $0.37 \pm 0.03$ & $15 \pm 0.2$ & $0.5 \pm 0.1$ & $\mathrm{a}$ & 0.37 & 1.4 \\
\hline & 327 & $1320 \pm 74$ & $0.27 \pm 0.07$ & $31 \pm 14$ & $0.7 \pm 0.1$ & & 0.26 & 5.6 \\
\hline & 74 & & $>0.15 \pm 0.06$ & $>100$ & 0.4 & $\mathrm{~b}$ & 0.11 & 147 \\
\hline \multirow[t]{3}{*}{$1508+55$} & 610 & & $>0.11$ & $>424$ & - & $\mathrm{c}$ & 0.43 & 0.4 \\
\hline & 327 & $74 \pm 15$ & - & - & - & & 0.31 & 1.6 \\
\hline & 74 & & $0.28 \pm 0.09$ & $47 \pm 37$ & 0.8 & $\mathrm{~b}$ & 0.13 & 42 \\
\hline
\end{tabular}

Notes - a: the measured values are from S2000; b: the measured values from GRC93; c: the measured values from Kaspi \& Stinebring (1992).

Table 2. Known DISS parameters at $327 \mathrm{MHz}$ (Baht et al. 1999a) and some other parameters.

\begin{tabular}{ccccccccc}
\hline \hline & & & & & \multicolumn{4}{c}{ DISS parameters at 327 MHz } \\
\cline { 7 - 10 } PSR B & DM & Distance & $V_{\text {prop }}$ & $S_{327}$ & $\Delta v_{\mathrm{d}}$ & $\tau_{\mathrm{d}}$ & $V_{\text {iss }}$ & $\log C_{\mathrm{Ne}}^{2}$ \\
\hline $0329+54$ & 26.4 & $1.43^{a}$ & 145 & $1230 \pm 38$ & 0.131 & 274 & 186 & -4.11 \\
$1508+55$ & 19.6 & $0.99^{b}$ & $500^{c}$ & $60 \pm 2$ & 0.187 & 144 & 451 & -4.41 \\
\hline
\end{tabular}

Notes $-{ }^{a}$ the distance estimate is based on the electron density distribution given by Taylor \& Cordes (1993); ${ }^{b}$ the distance estimate is based on the electron density distribution given by Cordes $\&$ Lazio $(2002) ;{ }^{c}$ the value is calculated by using the updated proper motion measurement (Hobbs et al. 2004).

In Table 2, we provide the pulsars, their flux densities and DISS parameters at $327 \mathrm{MHz}$ along with their dispersion measures, distances, proper-motion speeds. The flux densities and the DISS parameters (i.e. de-correlation bandwidths $\Delta v_{\mathrm{d}}$, diffractive time scales $\tau_{\mathrm{d}}$, scintillation velocities $V_{\text {iss }}$, and scattering strength $C_{\mathrm{Ne}}^{2}$ ) at $327 \mathrm{MHz}$ are taken from Bhat et al. (1999a).

Bhat et al. (1999a) monitored the dynamic spectra of the two pulsars at $327 \mathrm{MHz}$ at about 10 epochs over 60 to 70 days. According to their observations, the de-correlation bandwidths and diffractive time scales of PSR B0329+54 vary from $0.04 \mathrm{MHz}$ to $0.3 \mathrm{MHz}$ and from $204 \mathrm{~s}$ to $400 \mathrm{~s}$ due to refractive effects and the mean values of the two parameters are $0.131 \mathrm{MHz}$ and $274 \mathrm{~s}$ respectively. For PSR B1508+55, they range from $0.1 \mathrm{MHz}$ to $0.29 \mathrm{MHz}$ and from $100 \mathrm{~s}$ to $180 \mathrm{~s}$, on the average values of $0.187 \mathrm{MHz}$ and $144 \mathrm{~s}$, respectively. Comparing with the ranges of the de-correlation bandwidths, our observational bandwidth of $2 \mathrm{MHz}$ at $327 \mathrm{MHz}$ is large enough to avoid the diffractive scintillation confusing the observational results.

We use the mean values of the diffractive parameters observed at $327 \mathrm{MHz}$ (in Table 2) to calculate the predicted refractive scintillation parameters of these pulsars at 610, 327 and $74 \mathrm{MHz}$ using the scintillation theory. Table 1 gives the predicted values of modulation indices and refractive time scales at the three frequencies.

The predicted modulation indices $m_{\mathrm{r}, \mathrm{p}}$ are estimated using the expression

$m_{\mathrm{r}, \mathrm{p}} \approx 1.10\left(\frac{\Delta v_{\mathrm{d}}}{v_{\mathrm{obs}}}\right)^{0.17}$, valid to first order of a Kolmogorov spectrum for a statistically homogeneous medium with the inner scale less than the field coherent scale (Romani et al. 1986), where $\Delta v_{\mathrm{d}}$ is the decorrelation bandwidth of diffractive scintillation in $\mathrm{MHz}, v_{\mathrm{obs}}$ is the central frequency of observation in $\mathrm{MHz}$. To predict the refractive parameters at 74 and $610 \mathrm{MHz}$, we use frequencyscaling laws $\Delta v_{\mathrm{d}} \propto v_{\mathrm{obs}}^{4.4}$ and $\tau_{\mathrm{d}} \propto v_{\mathrm{obs}}^{1.2}$ to derive $\Delta v_{\mathrm{d}}$ and $\tau_{\mathrm{d}}$ at these frequencies (Romani et al. 1986).

We predict the refractive time scales by using the equation (GRC93),

$T_{\mathrm{r}, \mathrm{p}} \approx 0.0011 \lambda_{\mathrm{obs}}^{2.2} D^{0.5} v_{\Delta v_{\mathrm{d}}}^{2.2} \Delta v_{\mathrm{d}}^{-0.5} V_{\mathrm{iss}}^{-1}$

valid for an extended medium model and a Kolmogorov spectrum with the inner scale less than the field coherence scale (Rickett et al. 1984; Coles et al. 1987), where $\lambda_{\text {obs }}$ is the observational wavelength in meters, $D$ is the distance from pulsar to observer in kpc, $\Delta v_{\mathrm{d}}$ is the de-correlation bandwidth of diffractive scintillation in $\mathrm{MHz}, V_{\text {iss }}$ is the scintillation velocity in $\mathrm{km} \mathrm{s}^{-1}$, then $T_{\mathrm{r}, \mathrm{p}}$ is the predicted refractive timescales in days. This equation includes the frequency-scaling law described above to give the refractive time scale at observational wavelength $\lambda_{\mathrm{obs}}$ in terms of $\Delta v_{\mathrm{d}}$ observed at frequency $v_{\Delta v_{\mathrm{d}}}$ (in $\mathrm{MHz}$ ).

The logarithmic slope $\gamma$ of structure regime contains valuable information about the density inhomogeneity spectrum of the interstellar medium, and probes both the spectrum of the ionized medium and its distribution along the line of sight. The index $\gamma$ is sensitive to the detailed distribution of the scattering material (Simrnova et al. 1998; Cordes \& Rickett 1998). The formalism developed in Goodman \& Narayan (1985) and Romani et al. (1986) indicates $\gamma=\beta-3$ for an extended 
medium with a Kolmogorov spectrum (Coles 1988; Shishov 1993). Thus the expected value of $\gamma$ for an extended distribution of scattering material with a Kolmogorov spectrum is 0.67 . By contrast, for scattering material confined to a thin screen and obey a Kolmogorov spectrum, the slope value is $\gamma=2.0$ (e.g., Romani et al. 1986; Coles 1988). We now discuss the results for each pulsar.

\subsection{PSR B0329+54}

At all of the three frequencies, the measured modulation indices of the pulsar are in fair agreement with the predicted values calculated using refractive scintillation theory for a statistically homogeneous medium with a Kolmogorov spectrum. This pulsar has a long diffractive time scale at $327 \mathrm{MHz}$ and a correspondingly short predicted refractive time scales at the three frequencies. However, the measured time scales are much longer than that predicted by refractive scintillation theory. The predicted time scales at 327 and $610 \mathrm{MHz}$ are shorter than the minimum observable time scales of both observations. If the predicted refractive time scale were true, both observations at 327 and $610 \mathrm{MHz}$ would not be expected to be able to observe them. As noted by S2000, the observed time scales may be the real refractive time scales of the pulsar, and the current refractive theory is poor in describing the refractive time scale of the pulsar at least at the two higher frequencies. The logarithmic slopes $\gamma$ of the structure functions at all three frequencies rule out the possible screen geometry for scattering material distribution, which predicts much steeper slopes than observed, and they are consistent with a Kolmogorov spectrum and an extended distribution of scattering material.

\subsection{PSR $B 1508+55$}

Based on the electron density distribution given by Taylor \& Cordes (1993), the DM value of the pulsar gives a distance of $1.94 \mathrm{kpc}$. In our prediction, we adopted a new distance of $0.99 \mathrm{kpc}$ estimated by using the electron density distribution given by Cordes \& Lazio (2002). The pulsar has a large scintillation velocity measured at $327 \mathrm{MHz}$ (Bhat et al. 1999a), which is in good agreement with the proper motion velocity calculated by using the new distance and the updated proper motion measurement (Hobbs et al. 2004). Correspondingly, it has quite short predicted refractive time scales. The predicted time scales at $327 \mathrm{MHz}$ and $610 \mathrm{MHz}$ are less than the minimum observable time scales of the observations at the two frequencies. The observed refractive time scale at $74 \mathrm{MHz}$ is consistent with the predicted value for an extended medium model with a Kolmogorov spectrum. From the result of one year of observations at $610 \mathrm{MHz}$, Kaspi \& Stinebring (1992) suggest that the pulsar has a very long refractive time scale of more than 424 days at $610 \mathrm{MHz}$, which is much longer than the prediction for this pulsar. However, our observations at $327 \mathrm{MHz}$ did not show the long time-scale modulation suggested by Kaspi $\&$ Stinebring (1992). The logarithmic slope $\gamma$ of the structure function of the pulsar at $74 \mathrm{MHz}$ is 0.8 , much smaller than $\gamma=2.0$ suggested by the thin screen model of a scattering medium with a Kolmogorov spectrum.

\section{Discussion and conclusions}

The comparison between structure-function observations and theory is crucial to test the theory and to understand the properties and distribution of the interstellar scattering medium. However, there are two major aspects that affect the theoretic predictions. One is the validity of the theory itself which is the major object to be tested, another is the observed diffractive scintillation parameters that are chosen to do the quantitive predictions. Most of the published diffractive scintillation parameters have large uncertainties that make the predictions of refractive scintillation parameters unreliable. Thus, the predictions have always been regarded as order-of-magnitude estimates. More attention should be paid to determining accurate DISS parameters of pulsars for theoretical predictions of refractive properties.

Recently, Bhat et al. (1999a) monitored dynamic spectra of 18 pulsars at $10-90$ epochs over 100 to 1000 days and found large-amplitude fluctuations in quantities such as decorrelation bandwidths, scintillation timescales, and flux densities of the pulsars. They obtained more accurate and reliable estimates of diffractive scintillation parameters than those from the earlier observations by averaging out the fluctuations due to refractive scintillation effects. Our predicted refractive scintillation parameters are made reliable by using these measurements in the calculations. We should note that, comparing with the de-correlation band widths observed by Bhat et al. (1999) at $327 \mathrm{MHz}$, our observational bandwidth of $2 \mathrm{MHz}$ at $327 \mathrm{MHz}$ is large enough to avoid our observational results being contaminated by DISS effects.

As shown in Fig. 2, there is a clear frequency dependence of $T_{\mathrm{r}}$ and $m_{\mathrm{r}}$ of PSR B0329+54 according to the observational results at the three frequencies. The RISS theory predicts $m_{\mathrm{r}} \propto v^{0.578}$ for a Kolmogorov spectrum of scattering medium and $m_{\mathrm{r}} \propto v^{1.5}$ for steeper spectrum $\beta=4$ (Romani et al. 1986). The RISS theory gives $T_{\mathrm{r}} \propto v^{-2.2}$ for $\beta=11 / 3, T_{\mathrm{r}} \propto v^{-2}$ for $\beta=4$ and $T_{\mathrm{r}} \propto v^{-2.35}$ for $\beta=4.3$ (Stinebring \& Condon 1990). Fitting slopes for the $T_{\mathrm{r}}$-frequency dependence (right plot in Fig. 2) shows that all three predicted slopes of the frequency dependence of $T_{\mathrm{r}}$ are steeper than that of the observations, indicating the discrepancy between the observational results and the RISS theoretical predictions. Fitting slopes to the observed points on an $m_{\mathrm{r}}$-frequency plot (left log-log plot in Fig. 2) yields a slope of $0.6 \pm 0.3$, suggesting Kolmogorov turbulence of scattering material along line of sight of the pulsar.

The long-term flux density modulation of PSR B0329+54 at three frequencies is best described by a Kolmogorov power spectrum $(\beta=11 / 3)$ for electron density inhomogeneities in the interstellar medium, although the refractive time scales observed cannot be well predicted by current RISS theory. However, S2000 suggests that the observed modulation index of PSR B0329+54 at $610 \mathrm{MHz}$ can only be correctly predicted by introducing an inner scale of $10^{10} \mathrm{~cm}$ for a Kolmogorov spectrum electron density fluctuation. Their relatively small modulation index predicted by a Kolmogorov spectrum for 

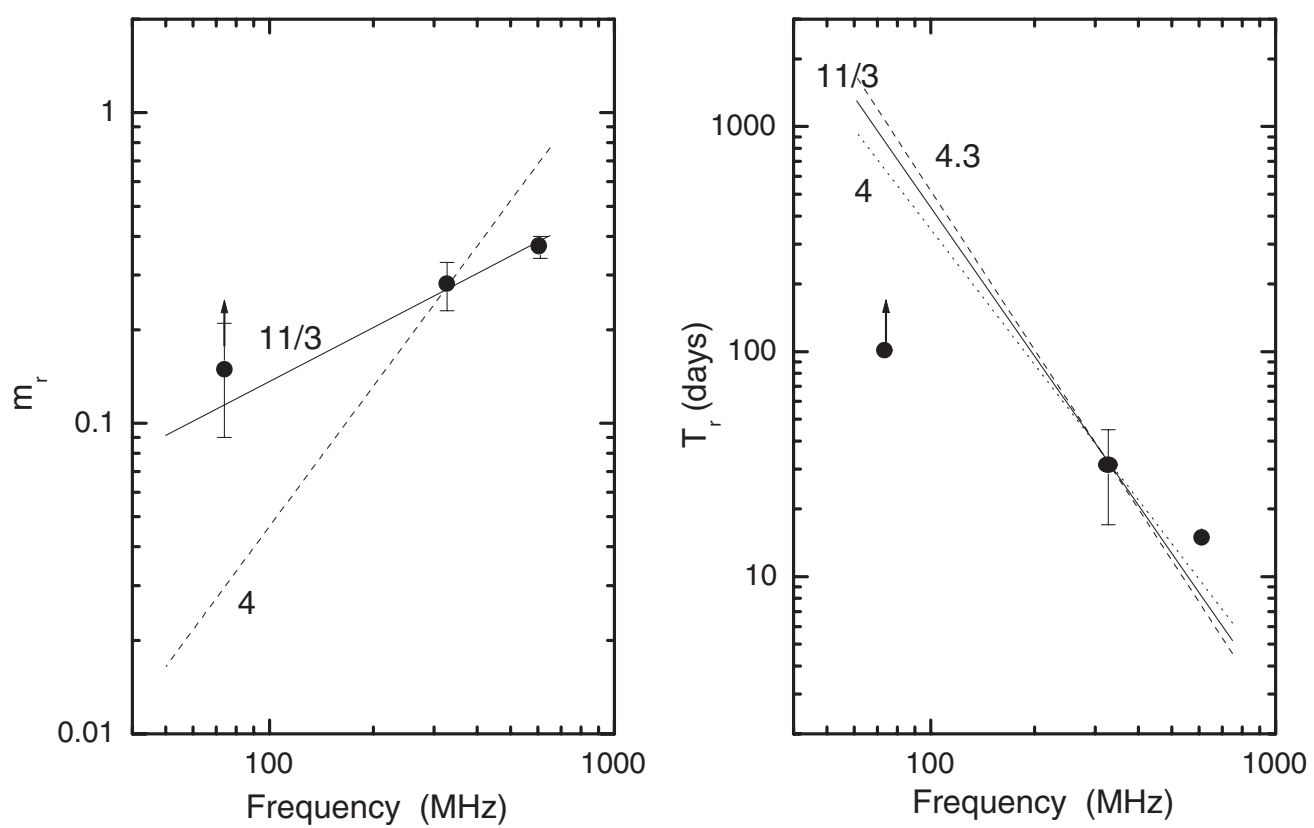

Fig. 2. Frequency dependence of modulation indices $m_{\mathrm{r}}$ (left diagram) and refractive time scales $T_{\mathrm{r}}$ (right diagram) for PSR B0329+54. The solid and dashed lines in the left diagram show the frequency dependent slopes of $m_{\mathrm{r}}$ predicted for electron density inhomogeneity spectrum indices $\beta=11 / 3$, and $\beta=4$ respectively. The solid, dashed and dot lines in right diagram present the theoretical predictions of frequency dependence of $T_{\mathrm{r}}$ for $\beta=11 / 3, \beta=4$ and $\beta=4.3$, respectively.

extended medium with a small inner scale may be due to the small diffractive de-correlation bandwidth they used for the prediction. The modest flux density modulation of PSR $\mathrm{B} 1508+55$ at three frequencies suggests that the electron density fluctuations have a power spectrum exponent $\beta<4$ along line of sight of the pulsar. The structure functions of both pulsars indicate the extended distribution of scattering medium along the lines of sight.

Although the observations at all three frequencies were sensitive to enhanced refractive scintillation events that support a steep spectrum model or the inner scale model, all the observations have not reported intensity spikes in pulsar flux densities or dramatic intensity fluctuations noted by early observations for other pulsars (Cole et al. 1970; Helfand et al. 1977; Fiedler et al. 1987). Thus we do not need to introduce an enhanced refractive scintillation mechanism to explain the observations presented in this paper.

Acknowledgements. This work was supported by National Natural Science Foundation of China.

\section{References}

Armstrong, J. W., Rickett, B. J., \& Spangler, S. R. 1995, ApJ, 443, 209

Bhat, N. D. R., Rao, A. P., \& Gupta, Y. 1999a, ApJS, 121, 483

Bhat, N. D. R., Rao, A. P., \& Gupta, Y. 1999b, ApJ, 514, 249

Bhat, N. D. R., Rao, A. P., \& Gupta, Y. 1999c, ApJ, 514, 272

Blandford, R. D., \& Narayan, R. 1985, MNRAS, 213, 591

Cole, T. W., Hesse, H. K., \& Page, C. G. 1970, Nature, 225, 712
Coles, W. A. 1988, Proceedings Radio wave Scattering in the Intersteller Medium, AIP Conf. Ser., 174, 163

Coles, W. A., Frehlich, R. G., Rickett, B. J., \& Codna, J. L. 1987, ApJ, 315,666

Cordes, J. M., \& Lazio, T. J. W. 2002 [arXiv:astro-ph/0207156]

Cordes, J. M., \& Rickett, B. J. 1998, ApJ, 507, 846

Cordes, J. M., \& Wolszczan, A. 1986, ApJ, 307, L27

Fiedler, R. L., Dennision, B., Johnston, K. J., \& Hewish, A. 1987, Nature, 326, 675

Goodman, J., \& Narayan, R. 1985, MNRAS, 214, 519

Gupta, Y., Rickett, B. J., \& Coles, W. A. 1993, ApJ, 403, 183 (GRC93)

Helfand, D. J., Fowler, L. A., \& Kuhlman, J. V. 1977, AJ, 82, 710

Hobbs, G., Faulkner, A., Stairs, I. H., et al. 2004, MNRAS, 352, 1439

Kaspi, V. M., \& Stinebring, D. R. 1992, AJ, 392, 530

LaBrecque, D. R., Rankin, J. M., \& Cordes, J. M. 1994, AJ, 108, 1845

Lambert, H. C., \& Rickett, B. J. 1999, ApJ, 517, 299

Rickett, B. J. 1990, ARA\&A, 28, 561

Rickett, B. J., \& Lyne, A. G. 1990, MNRAS, 244, 68

Rickett, B. J., Coles, W. A., \& Bourgois, G. 1984, A\&A, 134, 390

Rickett, B. J., Lyne, A. G., \& Gupta, Y. 1997, MNRAS, 287, 739

Romani, R. W., Narayan, R., \& Blandford, R. 1986, MNRAS, 220, 19

Shishov, V. I. 1993, Astron. Rep., 37, 378

Sieber, W. 1982, A\&A, 113, 311

Simrnova, T. V., Shishov, V. M., \& Stinebring, D. R. 1998, Astron. Rep., 42, 766

Stinebring, D. R., \& Condon, J. J. 1990, AJ, 352, 207

Stinebring, D. R., Smirnova, T. V., Hankins, T. H., et al. 2000, ApJ, 539, 300 (S2000)

Tayor, J. H., \& Cordes, J. M. 1993, ApJ, 411, 674

Zhou, A. Z., Wu, X. J., \& Esamdin, A. 2003a, A\&A, 403, 1059

Zhou, A. Z., Wu, X. J., \& Esamdin, A. 2003b, Chin. Phys. Lett., 20, 1405 